\title{
Using growth hormone as an adjuvant in IVF: Live birth outcomes from various poor prognosis scenarios
}

\author{
John L Yovich 1, 2, ${ }^{*}$, Shanthi Srinivasan ${ }^{1}$, Mark Sillender ${ }^{1}$, Shipra Gaur ${ }^{1}$, Philip Rowlands ${ }^{1}$ and Peter M \\ Hinchliffe ${ }^{1}$ \\ 1 PIVET Medical Centre Perth, Western Australia Australia 6007. \\ 2 Department of Pharmacy and Biomedical Sciences Curtin University Perth, Western Australia Australia 6845.
}

GSC Biological and Pharmaceutical Sciences, 2021, 15(01), 063-080

Publication history: Received on 13 February 2021; revised on 16 March 2021; accepted on 18 March 2021

Article DOI: https://doi.org/10.30574/gscbps.2021.15.1.0083

\begin{abstract}
Following 5 recent studies at PIVET several female factors were defined which enabled the clear categorization for a poor prognosis in IVF, namely advanced female age $\geq 42$ years, very low antral follicle count (AFC $<5)$, very low serum anti-Mullerian hormone level (AMH $<5 \mathrm{pmol} / \mathrm{L})$, serum Insulin growth factor-1 (IGF-1 level) in the lowest quartile, repetitive failed IVF cycles $(\geq 3)$ and the failure of residual embryos to undergo cryopreservation. Following an Assessment Cycle (AC) to define the first 4 factors in IVF-naïve women, women were offered recombinant growth hormone $(\mathrm{rGH})$ as an adjuvant at $1.0 \mathrm{IU}$ daily for 6 weeks in the lead-up to the oocyte pick-up of their first IVF treatment cycle. Of 1173 women who proceeded directly into IVF after completing an AC, 252 women (21.5\%) utilized rGH initiating 426 IVF cycles. Very low AFC and AMH levels were defined in 51 of the women who proceeded through 90 IVF treatment cycles utilizing rGH. Clinical outcomes included cancellation rates (reduced among rGH users, $\mathrm{p}<0.01$ ), oocytes retrieved (no significant benefit from $\mathrm{rGH}$ ), oocyte utilization (apparent benefit for rGH in older women with several factors), significant improvement in embryo utilization rates for older women with several factors (incremental cycles $\geq 3$; $p<0.002)$ or failure to achieve cryopreserved embryos $(\mathrm{p}<0.02)$. However, these benefits failed to translate into an improved pregnancy or live birth productivity rate nor a reduction in miscarriage rates; partly due to the low numbers of women with several poor prognosis factors. Furthermore, a note of caution emerged from this study as younger women who did not receive rGH had significantly better live birth outcomes ( $\mathrm{p}<0.0001$ from initiated cycles), regardless of the number of poor prognosis factors identified. Nonetheless, we encourage prospective studies to continue, focusing only on older women $\geq 40$ years with low ovarian reserve and additional poor prognosis factors.
\end{abstract}

Keywords: Growth Hormone (GH); Insulin growth factor-1 (IGF-1); In vitro fertilization (IVF); Assisted Reproductive Technology (ART); Pregnancy Productivity Rate; Live Birth Productivity Rate; Poor prognosis Fertility Factors.

\section{Introduction}

From a slow beginning in the 1970's, the generation of children from infertility settings applying Assisted Reproductive Technologies (ART) has now likely passed 10 million. Whilst this is hailed as a wonderful achievement, the reality is that the success rate per initiated treatment cycle is excellent for women under 35 years, but only mediocre for those aged between 35-40 years. For women $>40$ years, the results are quite poor and extremely low for those women $\geq 45$ years trying with autologous (self-generated) oocytes [1]. The data from the annual Australia and New Zealand Assisted Reproduction Database (ANZARD) shows that the majority of women treated in ART clinics during 2018 were aged between $35-40$ years, and $25.4 \%$ were aged $>40$ years (concomitantly, $31.6 \%$ of male partners were aged $>40$ years). The ANZARD report of 2020 shows that 91\% of embryo transfers were conducted as a single embryo transfer (SET) and $57.2 \%$ were thaw cycles (where the vast majority involved blastocysts vitrified at the blastocyst stage). Of the

\footnotetext{
${ }^{*}$ Corresponding author: John L Yovich

PIVET Medical Centre Perth, Western Australia Australia 6007.
} 
numerous factors influencing the outcome of autologous treatment cycles, the woman's age was shown to be the main key factor. For a single initiated autologous ART cycle, the live birth rate per embryo transfer was $40.4 \%$ for women $<30$ years; $33.7 \%$ for those women aged $30-34$ years; $24.8 \%$ for those women aged 35-39 years; $9.5 \%$ for those women aged $40-44$ years; and only $0.8 \%$ for those women aged $\geq 45$ years. Of interest the live birth rates were a little higher from the cryopreserved embryos across the age ranges, rising to $7.8 \%$ for those women aged $\geq 45$ years, bearing in mind that many of these embryos were generated whilst the women were at a younger age.

Although age of the woman is the key factor causing a poor prognosis, our group has reported on five studies examining a range of additional identifiable factors. These studies are summarized:

- The IGF serum profile (Insulin growth factor-1; IGF-1, Insulin growth factor binding protein -3; IGFBP-3 \& the IGF Ratio; being IGFBP-3/IGF-1) was examined against clinical parameters, namely the woman's age as well as her stature and BMI [2]. A significant inverse reduction was shown in IGF-1 levels across the four age groups ( $<35$ years, $35-39$ years, $40-44$ years and $\geq 45$ years). Although there was no variation in IGFBP-3 levels, the IGF Ratio showed a significant progressive linear elevation with advancing age. With respect to both BMI and stature, none of the IGF profile parameters showed any variation.

- $\quad$ The IGF profile was examined against measures of the ovarian reserve, namely the antral follicle count (AFC) and the serum anti-Mullerian hormone level (AMH) [3]. The AFC shows highly significant sequential changes, from high to low, across the 4 age groups. Similarly, AMH also showed highly significant sequential changes, from high to low, across the 4 age groups. Furthermore, there was high concordance between AFC and AMH groupings with correlation $r=0.79$ for precise matching and $r=0.95$ when neighboring groups were included. However, IGF profiles did not vary across the entire range of AFC nor AMH measures.

- The measurement of serum human growth hormone (hGH) in women undertaking ART appears to have limited value [4] with only $12 \%$ of women recording $\geq 3.0 \mathrm{ng} / \mathrm{mL}$ on morning samples, the level which excludes Adult Growth Hormone Deficiency (AGHD). Furthermore, 22\% of samples recorded extremely low, close to the detection point of the assay. There was no correlation between hGH levels nor any aspect of the IGF profile, neither IGF-1 levels nor IGFBP-3 levels, neither the IGF Ratio. Additionally, hGH levels were not different among the clinical parameters of stature or BMI; nor for ovarian reserve parameters AMH or AFC.

- When recombinant $\mathrm{GH}(\mathrm{rGH})$ was given to women as an adjuvant in ART, significant effects were shown on the IGF profile [5]. In particular, the IGF-1 levels showed highly significant elevations, as did as its main binding protein, IGFBP-3, albeit to a lesser degree. Consequently, the IGF ratio, sometimes considered a more reliable marker of IGF-1 activity, was shown to be significantly reduced towards the normal range.

- The IGF profile showed significant prognostic value in the ART program with higher oocyte numbers retrieved at oocyte pick-up (OPU), and more of the oocytes were at the mature (metaphase; MII) stage. Consequently, a higher oocyte utilization rate was shown for those women whose IGF-1 levels were in the highest quartile [6]. Although the embryo utilization rates were similar across the IGF-1 quartiles, the implantation rate, reflected by the pregnancy rate, was higher when the productivity rate (being the cumulative effects of fresh and frozen embryos transferred from a single OPU) was measured. Finally, without any differences in miscarriage rates across the IGF-1 quartiles, the live birth productivity rate was also shown to be significantly greatest for those women with IGF-1 levels in the highest quartile.

These 5 studies set the background to determine whether rGH adjuvant could improve the clinical prognosis from ART where poor prognosis factors could be identified [7]. In this study, ART embraces treatments by in-vitro fertilization (IVF) and intra-cytoplasmic sperm injection (ICSI) along with embryo transfers (ETs) from fresh procedures as well as from those cryopreserved embryos arising from the same OPU.

\section{Material and methods}

\subsection{Study setting}

Following a 4-year period of training in London during the years 1976-1980, lead author JLY established the PIVET program for ART in Perth, Australia in 1981 [8,9], initially recording all treatment cycles and clinical outcomes in handwritten registers. From 2001, all ART treatments, numbering 23,509 have been comprehensively recorded in an internal validated data base using Filemaker Pro as well as providing data to ANZARD which publishes an annual report, available to the public [1]. Recently, as an Australian Government initiative, ANZARD also provides data to the YourIVFSuccess website, enabling an open disclosure of results from each and every of 90 participating IVF Clinics around Australia [10]. Since January 2011, the ART program at PIVET has been characterized by three important developments, firstly, encouraging amenable ART-naïve women to undertake a preliminary Assessment Cycle (AC) [11]; 
secondly, utilizing the well described PIVET dosing-algorithms for ovarian stimulation [12,13,14], one of which is shown in Table 1; and thirdly, a commitment to single embryo transfer (SET) procedures for both fresh and frozen embryos across the entire age profile. Currently, in keeping with a widely encouraged practice across Australia and New Zealand, SET procedures at PIVET is currently at $91 \%$ of all cases [10]. In association with this, PIVET commits to a blastocyst culture system whenever $\geq 3$ embryos are progressing on a Day-3 laboratory inspection, currently occurring in $90 \%$ of in vitro fertilization (IVF) cases. This means that there is also a high commitment to cryopreservation, which at PIVET is conducted using the Cryotop vitrification technique [15].

Table 1 One of the PIVET FSH-dosing Algorithms which covers for 12.5 IU and 25 IU increments of FSH.

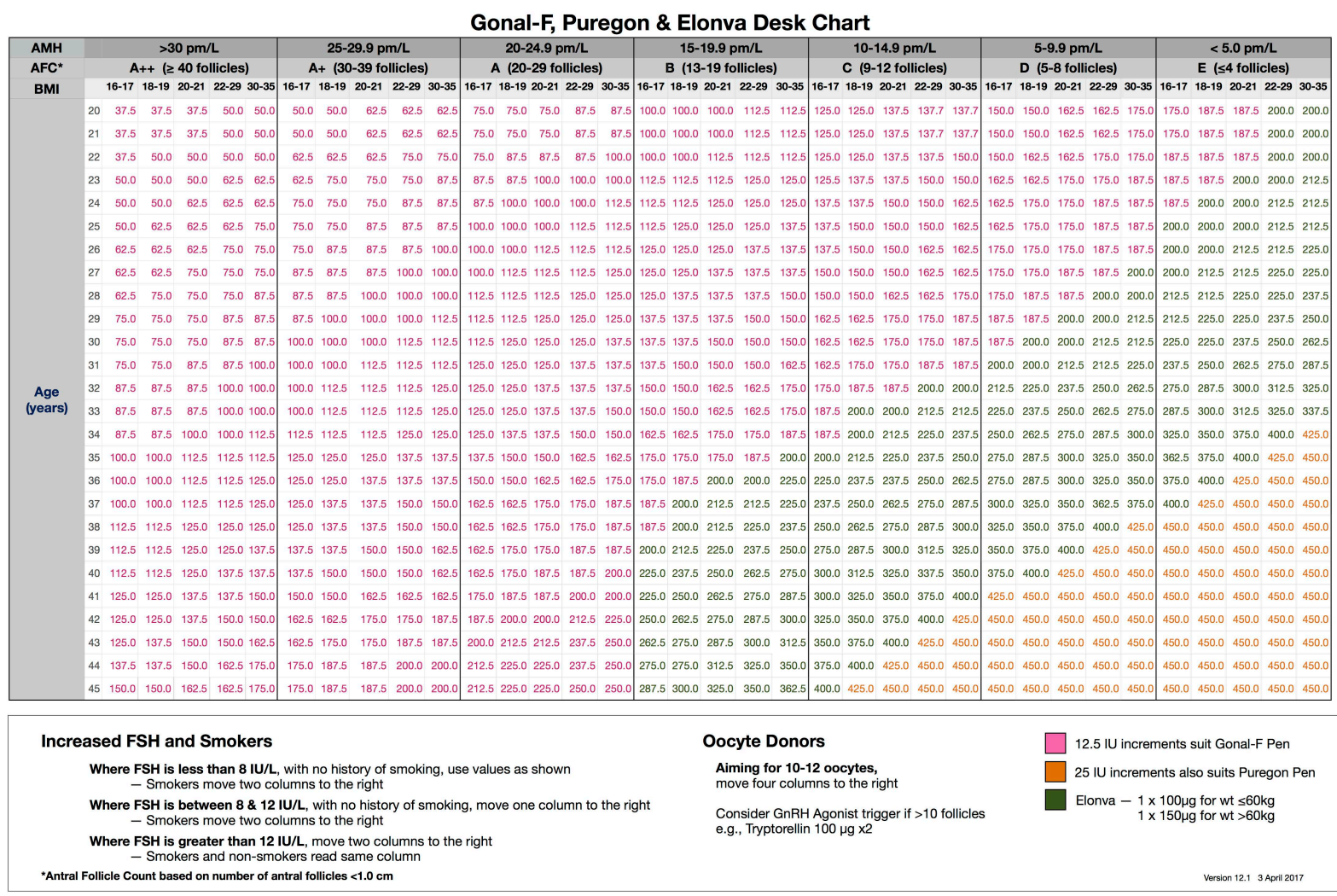

\subsection{Patient selection}

Across the period January 2011 to December 2019, 3751 women entered into 10,728 treatments of various ART categories. Figure 1 shows the derivation of 1633 women from a total 2319 women who had an AC which also included an insulin-like growth factor (IGF) profile comprising IGF-1 along with its main binding protein IGFBP-3, and a calculation of IGF ratio being the division of IGF-1 into the higher concentration IGFBP-3 level. In the current study we are exploring the relevance of a range of poor prognosis factors to the subsequent IVF treatment outcomes. From Figure 1 it can be seen that 1173 of the women who completed an AC with full IGF profile proceeded to a total 1761 IVF treatments, utilizing intra-cytoplasmic sperm injection (ICSI) according to well reported PIVET protocols where indicated [16]. This included an IVF-ICSI Split model for unexplained infertility cases. The poor prognosis criteria established from our former studies included advanced age of the woman $\geq 40$ years; a low ovarian reserve (PIVET Algorithm Group E indicating an AFC $<5$ antral follicles and AMH level $<5$ pmol/L [17]); an IGF-1 level in the lowest quartile ( $<21 \mathrm{nmol} / \mathrm{L}[2,6])$; incremental IVF treatment cycles ( $\geq 3$ OPU cycles [18]); and poor-quality embryos resulting in nil blastocysts available or suitable for cryopreservation (zero frozen) [19]. These factors have been analyzed alone and in combination.

\subsubsection{Patients utilizing rGH adjuvant therapy}

Women categorized as likely to have a poor prognosis were informed and offered access to one of 3 adjuvants, namely dehydroepiandrostenedione (DHEA), melatonin or $\mathrm{rGH}$ [11]. Most women elected to take their chances without adjuvants, or selected the least expensive options, namely DHEA or melatonin. However, 252 of the 1213 women $(20.8 \%)$ undertook a total of 426 IVF cycles using rGH adjuvant therapy (Figure 1). 


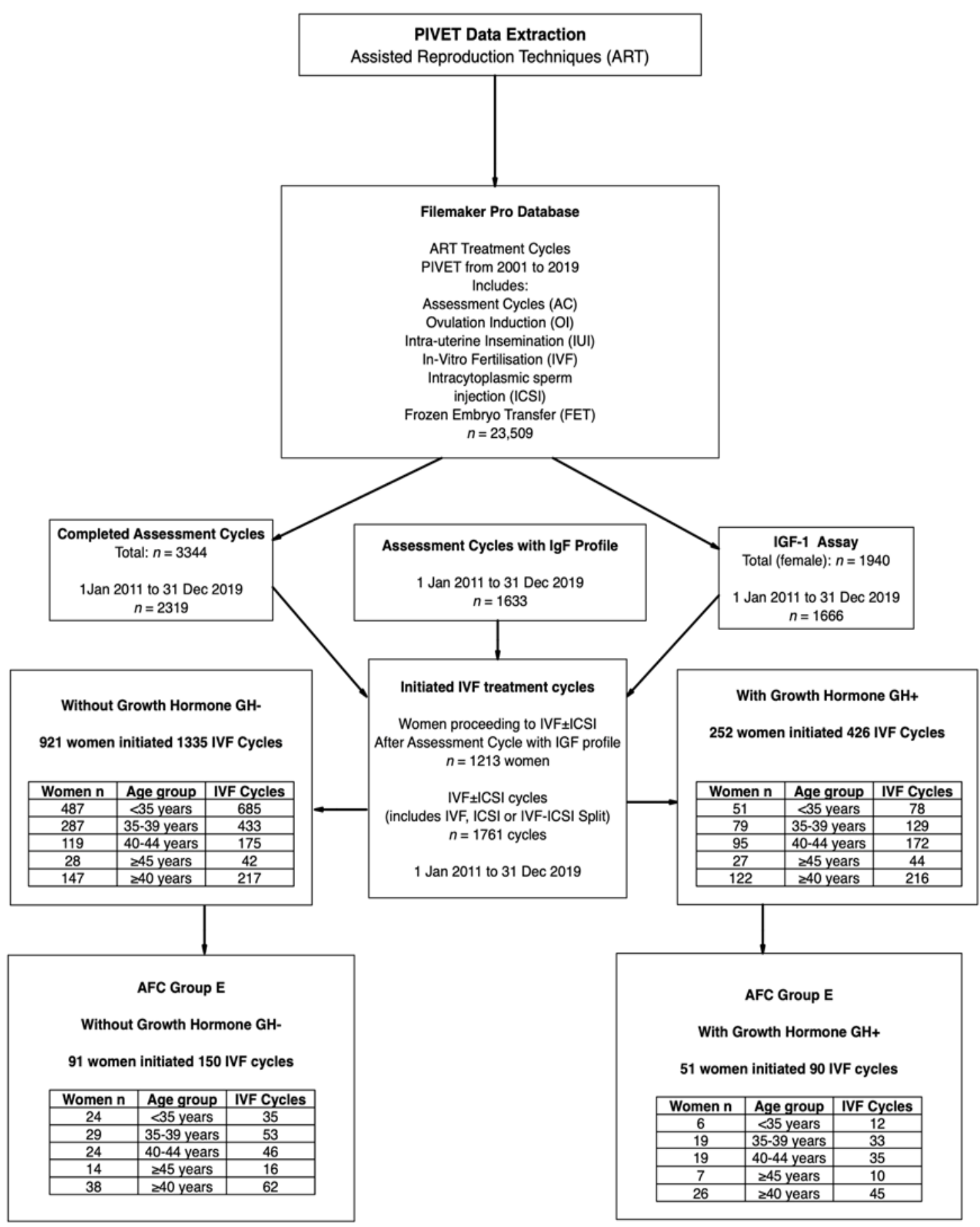

Figure 1 Flow diagram showing the derivation of the women who proceeded to IVF \pm rGH adjuvant treatment cycles after undertaking an assessment cycle.

The hGH regimen utilized SciTropin (SciGen, Belrose, Australia) $0.3 \mathrm{mg}$ self-injected subcutaneous daily beginning Day3 of the pre-IVF cycle for $\sim 45$ days leading up to the recombinant hCG (rhCG) Trigger injection (Merck Serono, Sydney, Australia) with patients receiving rGH at precisely $1.0 \mathrm{IU}$ per day prior to OPU. The information provided to the women is shown in Figure 2 which depicts the approved informed consent form signed by the women. A subset of 141 women with the lowest AFC (Group E) were analyzed according to their rGH use (51 women; 35.9\% undertook 90 IVF cycles; $37.5 \%$ ) and sub-classified for additional poor prognosis factors. 

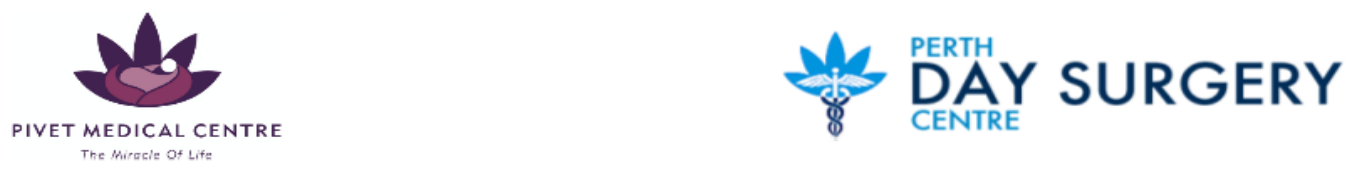

\section{PATIENT INFORMATION SHEET - GROWTH HORMONE}

\section{What is Growth Hormone?}

Growth hormone is a hormone secreted by the pituitary gland that stimulates growth, cell reproduction and cell regeneration. Current data analysis shows that biosynthetic Growth Hormone (SciTropin) is particularly effective for women who have produced poor quality embryos with few available for cryopreservation.

Approximately $35 \%$ of women do not achieve a livebirth from current standard IVF regimens and many seek adjustments, adjuvants and add-ons attempting to improve their prognosis. There are 10 areas of physiological focus and over 50 adjuncts are described (see Ref: 8 ). However, none have proven benefit according to the highest statistical requirements for evidence-based medicine (EBM). The brightest light among them is that for Growth Hormone, which has been the focus of study at PIVET for more than 12 years.

\section{Side Effects}

The studies (see references) involved 491 women who had a total 849 IVF cycles where GH adjuvant was used. No significant complications occurred to the women or their babies. Two women had mild swelling and joint pains in their hands, ceasing within a few days of stopping the $\mathrm{GH}$ injections.

\section{Cost of Growth Hormone}

Currently PIVET prescribes SciGen GH cartridges at $\$ 300$ each. The injections provide 1 IU per day (which is a normal physiological dose) for 15 days. Patients should discuss with their gynaecologist regarding regimens of 15 days, 30 days or 45 days (i.e. One, two or three cartridge course.)

\section{How is Growth Hormone Administered?}

The nurse will go through the process of how to administer Growth Hormone with you.

It is best to administer this medication at night time, as it works best with the body at night when it peaks naturally in Growth Hormone levels.

It Is important to avoid alcohol consumption during the Growth Hormone administration period.

Growth Hormone can be administered by two methods:

- 9 units daily by syringe - If you are given syringes please draw up 9 units daily

or

- 6 "clicks" by Omnitrope pen - load vial and dial up and administer 6 "clicks" (0.3) daily

\section{Blood Tests}

A baseline growth factors blood test will usually be obtained prior to starting Growth Hormone and then after each cartridge to assess the effect the medication is having on these factors. You may have already attended to this blood test during your initial testing. The nursing staff will advise and provide request forms when you require a blood test.

Reviewed by:
$\begin{aligned} & \text { Developed: June } 2018 \\ & \text { Nolumes/Groups/PIVET Documents/File \# } 18 \text { Info \& Consents/INFO SHEETS/207 Growth Hormone June } 2021\end{aligned}$
Page 1 of 2

Figure 2 The information and consenting form for those women planning to utilize rGH adjuvant treatment during their IVF cycle.

\subsection{IVF outcome parameters}

\subsubsection{Cycles initiated}

Cycles initiated denotes those autologous IVF cycles which commence ovarian stimulation with gonadotrophins on Day3. Approval requires that base-line parameters are met on day-2, namely serum gonadotrophins, follicle stimulating hormone (FSH) and luteinizing hormone (LH), indicating the woman is not menopausal or in ovarian failure. In addition, 
her ovarian hormones must be at basal levels, namely estradiol (E2) level $<150$ pmol/L and Progesterone (P4) level $<5$ nmol/L along with her pituitary Prolactin <750 IU/L [11].

\subsubsection{Cancelled cycle}

Cancelled cycle indicates the IVF cycle is abandoned, mostly prior to rhCG Trigger which is usually given around day12. This usually arises from a lack of response to the ovarian stimulation with lack of E2 elevation and failure to detect ovarian follicles $\geq 16 \mathrm{~mm}$ by transvaginal pelvic ultrasound. Cycles with poor responses are maximally stimulated with follicle stimulating hormone (FSH) at $450 \mathrm{IU} /$ day up to a maximum 16 days (day-19 of IVF cycle). Cycles may also be abandoned if there is evidence of premature ovulation (Rising LH, elevated P4 and marked fall in E2). This problem is unusual in current practice which routinely applies gonadotrophin releasing hormone (GnRH) antagonists [5]. Occasional causes of cancelled cycles may be patient-related issues such as intercurrent illness, domestic stresses and logistical problems related to travel from remote locations. Cases with zero oocytes recovered at OPU are also included in this category.

\subsubsection{Oocytes retrieved}

Oocytes retrieved denotes every oocyte detected following ovarian follicle aspiration at ovum pick-up (OPU). At PIVET this is undertaken by a single-lumen aspirating needle when follicle numbers $>12 \mathrm{~mm} \geq 5$; and a double-lumen flushing needle when there are fewer follicles.

\subsubsection{Oocyte utilization rate}

Oocyte utilization rate denotes the number of oocytes which fertilize and contribute to the formation of embryos which are utilized in a fresh embryo transfer (ET) procedure or a frozen embryo transfer (FET) procedure after a period of cryopreservation. This is factored for all oocytes, be they at MII, MI or the germinal vesicle stage, or even if they are subsequently shown to have degenerated or have fractured zona pellucidae. Specifically, this oocyte utilization rate is not the same as the oocyte fertilization rate which designates the number of embryos arising after oocytes are selected for insemination or ICSI; considered by us to be a less useful measure.

\subsubsection{Increased monitoring}

Increased monitoring relates to those women who have $>12$ oocytes recovered at OPU and are therefore classified as being at risk for ovarian hyperstimulation syndrome (OHSS). Such women have daily contact with PIVET to discuss the woman's wellbeing, including measurement of her abdominal girth, description of urine characteristics (light or dark color and output), as well as blood test monitoring every 2nd or 3rd day of the luteal phase, with concern if E2 $>6000 \mathrm{pm} / \mathrm{L}$ and/or P4 $>600 \mathrm{~nm} / \mathrm{L}$. Such patients may return to PIVET for IV fluids and specific gravity monitoring of their urine. Occasional women will need paracentesis of ascites and hospitalization, although such events have reduced to $0.1 \%$ in recent years.

\subsubsection{Embryo utilization rate}

Embryo utilization rate denotes the number of fertilized oocytes (defined at the two-pronuclear; 2PN stage), which are then utilized in a fresh ET procedure or subsequently in a FET procedure after a period of cryopreservation. PIVET protocols [11] means that $\sim 85 \%$ of all oocytes retrieved undergo a corona-cumulus stripping process for ICSI, which enables identification of the maturational stage of the oocyte, particularly the MII oocyte which has released a single polar body. Those oocytes subjected to IVF-only, have the maturational stage presumed at the 18-hour PN-stage check when pipette-stripping occurs. If the 2PN stage is identified, it is presumed that the oocyte must have been at the MII stage at OPU.

\subsubsection{Embryos frozen}

Embryos frozen denotes the proportion of all embryos generated which are cryopreserved by vitrification. This is usually those embryos which reach the blastocyst stage on day-5 or day- 6 with Gardner Grading at the level of 3BB or better, ideally 4AA or 5AA for best prognosis [19]. Sometimes all suitable embryos are cryopreserved in a "freeze-all" cycle to reduce the OHSS risk. The majority of IVF cycles in women classified as having a good prognosis can generate 3-4 high-grade embryos from 10 oocytes recovered at OPU, meaning that 2 or 3 will be cryopreserved after the SET procedure. 


\subsubsection{Freeze-all embryos}

Freeze-all embryos denotes those cases where a fresh embryo transfer is not performed, mainly to reduce the risk of OHSS and suitable embryos, mostly blastocysts, are committed to cryopreservation for future FETs. This option is considered when $\geq 15$ oocytes are recovered at OPU or fresh ETs are deferred for other reasons (including when the woman is unwell, or embryo transfer is deferred awaiting procedures such as hysteroscopy or laparoscopy to correct pelvic conditions).

\subsubsection{Pregnancy productivity rate}

Pregnancy productivity rate denotes the total number of pregnancies arising after both fresh ETs and FETs related to a single initiated cycle reaching the stage of OPU. This means the freeze-all or freeze-best embryo strategies do not prejudice the "pregnancy rate". It is for this reason we have developed such terminology rather than the oft-used cumulative pregnancy rate which, traditionally related to a number of IVF/OPU cycles [20]. Furthermore, the pregnancy productivity rate can be designated from the initiation of a treatment cycle (following the baseline blood test performed on cycle Day-2); from the stage of an OPU procedure where at least one oocyte is recovered; or from the stage of reaching an ET procedure (performed in either the fresh cycle or following cryopreservation). This parameter is different from the pregnancy productivity rate per each and every ET; considered by us to be a less useful measure.

\subsubsection{Miscarriage rate}

Miscarriage rate denotes those pregnancies which do not advance to a livebirth and are invariably lost before 20 weeks "gestation"; the division point for obstetric outcomes. At PIVET pregnancies are diagnosed provisionally at Day-19 of the luteal phase as "4-weeks" when serum BhCG is detected > 25 IU/l. At this stage the pregnancy is "biochemical only" but is tracked each week until a transvaginal pelvic scan at 7-weeks denotes the presence of an intra-uterine gestational sac, expectantly with a definable viable fetus. However, at PIVET clinical pregnancy is diagnosed at week-5 if the BHCG elevation is around 5 to 10 -fold and at week- 6 if there is a further 2 to 5 -fold rise along with associated appropriate levels of E2 and P4. PIVET has hormonal support strategies for those pregnancies with threatened miscarriage and suboptimal P4 levels [5]. Miscarriage rates include pregnancies of unknown location, ectopic gestations, blighted ovum losses and terminations of abnormal or demised fetuses prior to 20 weeks. In this study the miscarriage rate is given as all pregnancy losses (numerator) as a proportion of clinical pregnancies (denominator).

\subsubsection{Livebirth productivity rate}

Livebirth productivity rate denotes the total number of pregnancies arising after both fresh ETs and FETs related to a single initiated cycle reaching the stage of OPU and delivering after 20 weeks. As with pregnancy rates, this means freeze-all or freeze-best embryo strategies do not prejudice the "pregnancy rate". It is for this reason we have developed such terminology rather than the oft-used cumulative live birth rate which, traditionally related to a number of IVF/OPU cycles [20]. Each delivery is counted as one livebirth, even where twins or higher-order multiples are delivered. Stillbirths are not included as a live birth, but perinatal losses are included. Fortunately, such adverse outcomes are now uncommon in Australia due to the SET policy [11] and PIVET records twinning rates at under $1.0 \%$ with nil triplets or higher order multiples in recent years [10]. Furthermore, the live birth productivity rate can be designated from the initiation of a treatment cycle (following the baseline blood test performed on cycle Day-2); from the stage of an OPU procedure where at least one oocyte is recovered; or from the stage of reaching an ET procedure (performed in either the fresh cycle or following cryopreservation). This parameter is different from the live birth productivity rate per each and every ET; considered by us to be a less useful measure.

\subsection{Statistics}

Data extractions from the Filemaker database were placed in Microsoft Excel spreadsheets and sorted according to the relevant tests. Thereafter the sorted data was placed in the application Past 4.03 (developed by Øyvind Hammer) [21] for statistical data analysis. This application also generated the Tables comprising the statistical summaries, finally placed in Microsoft Word for clearer display. Having demonstrated that the data comprising the IGF profile (IGF-1, IGFBP-3 and IGF Ratio) are all distributed in a Normal fashion, the relationship among the means was examined by oneway ANOVA for overall comparison. The Kruskal-Wallis test was applied to examine equality between sample medians and Mann-Whitney applied for pairwise comparisons between individual sub-groups. Ratio comparisons between two groups were analyzed in 2x2 contingency tables, mainly by Fisher's exact test, or by Chi-squared applying Yates' continuity correction factor for the larger data sets. Following corrections, probability values of $p<0.01$ were considered significant for any test. As this data is retrospective by design, with wide variance and large kurtosis, hence several comparisons which were borderline, ranging 0.03 to 0.06 ; were classified as being of uncertain significance hence not 
claimed as significant. The Figures displayed for this study are derived from Excel v 16.42 (2020) and X-Diagram v 5.7 application (2021) developed by Vu Tien Thinh.

\section{Results}

Table 2 Clinical features and outcomes for all IVF treatment cycles $( \pm \mathrm{rGH}$ ) in IVF-naïve women who completed an Assessment Cycle 2011-2019.

\begin{tabular}{|c|c|c|c|c|c|c|c|c|c|c|c|c|}
\hline \multicolumn{13}{|c|}{ All IVF士ICSI cycles in IVF-naïve women who completed an Assessment Cycle 2011-2019 } \\
\hline Ages & \multicolumn{2}{|c|}{$<35$ yrs } & \multicolumn{2}{|c|}{$35-39$ yrs } & \multicolumn{2}{|c|}{$40-44$ yrs } & \multicolumn{2}{|c|}{ \pm 45 yrs } & \multicolumn{2}{|c|}{ All $\geq 40$ yrs } & \multicolumn{2}{|c|}{ Totals } \\
\hline \pm Growth $\mathrm{H}$ & GH- & $\mathrm{GH}+$ & GH- & $\mathrm{GH}+$ & GH- & $\mathrm{GH}+$ & GH- & GH+ & GH- & $\mathrm{GH}+$ & $\mathrm{sH}-$ & $\mathrm{GH}$ \\
\hline No. Cycles & 685 & 78 & & & & & & & & & & \\
\hline No. & & 51 & & 79 & & & & & & & & \\
\hline Can & & & & & & & & & & & & \\
\hline Ooc & & & & & & & & & & & & 2 \\
\hline & & & & & & & & & & & & \\
\hline & & & & & & & & & & & & \\
\hline & & & & & & & & & & & & \\
\hline Pres & & & & & & & & & & & & \\
\hline Preg. & 77 & & 57 & & & & & & & & & .0 \\
\hline Preg & 91 & 62 & 73 & & 35 & 20 & 26 & & 34 & 1 & .0 & 4.2 \\
\hline & & & & & & & & & & & & .0 \\
\hline & & $7 x$ & & & & & & & & & & .7 \\
\hline & & & & & & & & & & & & 7.3 \\
\hline L/B Prod \% / ET & 75.4 & 47.1 & 54.5 & 33.3 & 19.3 & 12.6 & 20.0 & 3.8 & 19.4 & 10.9 & 60.9 & 24.6 \\
\hline
\end{tabular}

The clinical outcomes for the 1173 IVF-naïve women who completed a total of 1761 autologous IVF \pm ICSI cycles following an AC are shown in Table 2 where the data is presented according to age groupings and the use of rGH cotreatment. The cancellation rates for the women who did not receive $\mathrm{rGH}$ ranged from $9.1 \%$ in the age group of 35-39 years to $26.2 \%$ for those women aged 45 years or more. Where rGH was used, the rates ranged from $2.6 \%$ in the youngest grouping ( $<35$ years) to $13.6 \%$ in the oldest grouping ( $>45$ years). Overall, the use of rGH co-treatment resulted in fewer cancellations $(10.7 \%$ vs $9.2 \%$; $<<0.01)$. Oocyte numbers recovered were higher in the younger women who did not receive $\mathrm{rGH}$, and a little higher in the oldest group ( $\geq 45$ years) but overall, there was no significant benefit seen from rGH. Similarly, oocyte utilization was slightly higher for women $\geq 40$ years, but overall, no significant benefit could be demonstrated for rGH co-treatment. Similarly, although embryo utilization was a little higher among women $\geq 40$ years receiving $\mathrm{rGH}$, no significant benefit could be demonstrated overall. Furthermore, the proportion of embryos cryopreserved across the age groups was never higher in the rGH adjuvant group. With respect to pregnancy outcomes, these were never higher according to $\mathrm{rGH}$ usage. The miscarriage rates appeared modestly reduced for women $\geq 40$ years using rGH (37.5\% vs $43.5 \%$ ), but overall, across all age groupings, no significant benefit could be demonstrated. The most relevant parameter, that of live birth productivity rates, was not improved across the age groups by adjuvant rGH usage.

Given the negative results for rGH shown in the overall group, the main study conducted here focused on the women categorized as having a poor prognosis, summarized in Table 3 and presented for detail and clarity in 11 Figures. The cancellation rates among the 142 women with very low ovarian reserve (AFC group E) who initiated 240 IVF treatment cycles are shown in Figure 3. 
Table 3 Clinical features and outcomes for all IVF treatment cycles ( $\pm r G H)$ in IVF-naïve women who completed an Assessment Cycle during 2011-2019 showing the lowest antral follicle count (Group E).

\begin{tabular}{|l|l|l|l|l|l|l|l|l|l|l|l|l|l|}
\hline All IVF \pm ICSI cycles in IVF-naïve women with AFC Group E who completed an AC 2011-2019 \\
\hline Ages & $<35$ yrs & $35-39$ & yrs & $40-44$ & yrs & \pm 45 & yrs & \multicolumn{2}{|c|}{ All $\geq 40$ yrs } & \multicolumn{2}{|c|}{ Totals } \\
\hline \pm Growth Hormone & GH- & GH+ & GH- & GH+ & GH- & GH+ & GH- & GH+ & GH- & GH+ & GH- & GH+ \\
\hline No. Cycles & 35 & 12 & 53 & 33 & 46 & 35 & 16 & 10 & 62 & 45 & 150 & 90 \\
\hline No. Women & 24 & 6 & 29 & 19 & 24 & 19 & 14 & 7 & 38 & 26 & 91 & 51 \\
\hline Cancellation \% & 8.6 & 8.3 & 17.0 & 15.2 & 21.7 & 28.6 & 18.8 & 10.0 & 21.0 & 24.4 & 16.7 & 18.9 \\
\hline Oocytes/OPU & 9.2 & 6.8 & 6.8 & 4.4 & 5.2 & 4.6 & 3.5 & 2.3 & 4.8 & 4.0 & 6.6 & 4.4 \\
\hline Oocyte Utiliz \% & 31.3 & 28.1 & 27.8 & 35.0 & 28.9 & 27.8 & 30.4 & 47.6 & 29.2 & 30.9 & 29.4 & 31.9 \\
\hline Embryo Utiliz \% & 55.4 & 50.0 & 49.1 & 57.3 & 59.3 & 66.7 & 42.4 & 100 & 54.8 & 72.4 & 52.9 & 60.9 \\
\hline Embryos Cryo \% & 36.4 & 19.4 & 27.1 & 18.4 & 19.4 & 25.0 & 20.0 & 20.0 & 19.5 & 24.2 & 28.5 & 20.8 \\
\hline Preg Prod \% / Init & 74.3 & 25.0 & 35.8 & 24.2 & 10.9 & 11.4 & 12.5 & 10.0 & 11.3 & 11.1 & 34.7 & 17.8 \\
\hline Preg Prod \% / OPU & 81.3 & 27.3 & 43.2 & 28.6 & 13.9 & 16.0 & 15.4 & 11.1 & 14.3 & 14.7 & 41.6 & 21.9 \\
\hline Preg Prod \% /ET & 92.9 & 42.9 & 59.4 & 38.1 & 18.5 & 30.8 & 33.3 & 20.0 & 21.2 & 27.8 & 55.9 & 34.8 \\
\hline Miscarriage \% & 15.4 & 66.7 & 21.1 & 37.5 & 20.0 & 50.0 & 0.0 & 0.0 & 14.3 & 40.0 & 17.3 & 43.8 \\
\hline L/B Prod \% / Init & 62.1 & 8.3 & 28.3 & 15.2 & 8.7 & 5.7 & 12.5 & 10.0 & 9.7 & 6.7 & 28.7 & 10.0 \\
\hline L/B Prod\% / OPU & 68.8 & 9.1 & 34.1 & 17.9 & 11.1 & 8.0 & 15.4 & 11.1 & 12.2 & 8.8 & 34.4 & 12.3 \\
\hline L/B Prod \% / ET & 78.6 & 14.3 & 46.9 & 23.8 & 14.8 & 15.4 & 33.3 & 20.0 & 18.2 & 16.7 & 46.2 & 19.6 \\
\hline
\end{tabular}

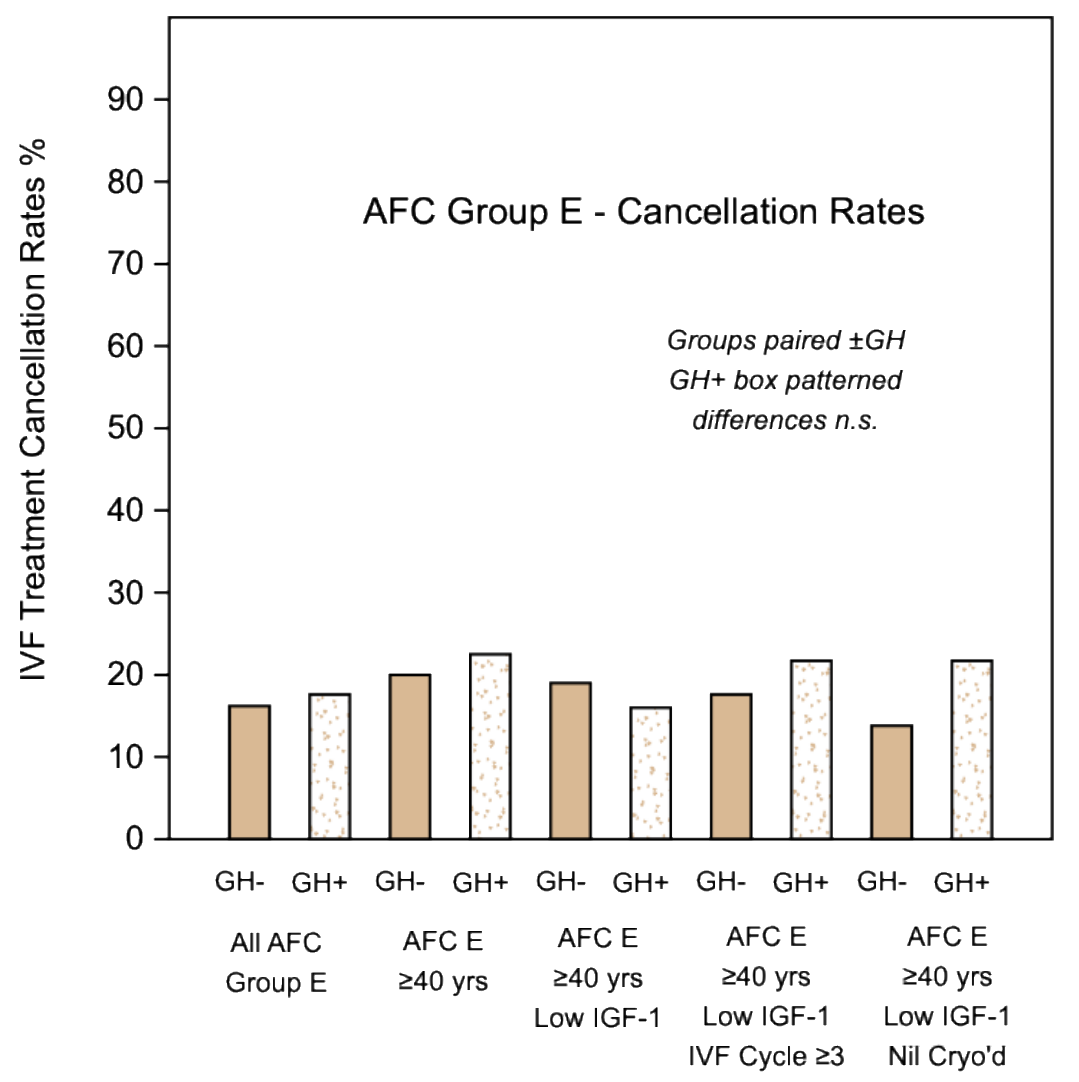

IVF Poor-prognosis Treatment Categories

Figure 3 Cancellation rates among women classified as poor prognosis who initiated IVF treatments \pm rGH 
For women not receiving $\mathrm{rGH}$ the rates ranged from $8.6 \%$ for young women $(<35$ years) to $21.0 \%$ for older women $(\geq 40$ years). For those receiving rGH the rates were not significantly lower, ranging from $8.3 \%$ for the younger to $24.4 \%$ for the older women. When addition poor prognosis factors were analyzed, including those women with IGF-1 levels in the lowest quartile, those women undertaking their third or more incremental cycle, and those cycles which did not achieve any cryopreserved embryos, no significant differences were detected between those who did, or did not receive, hGH.

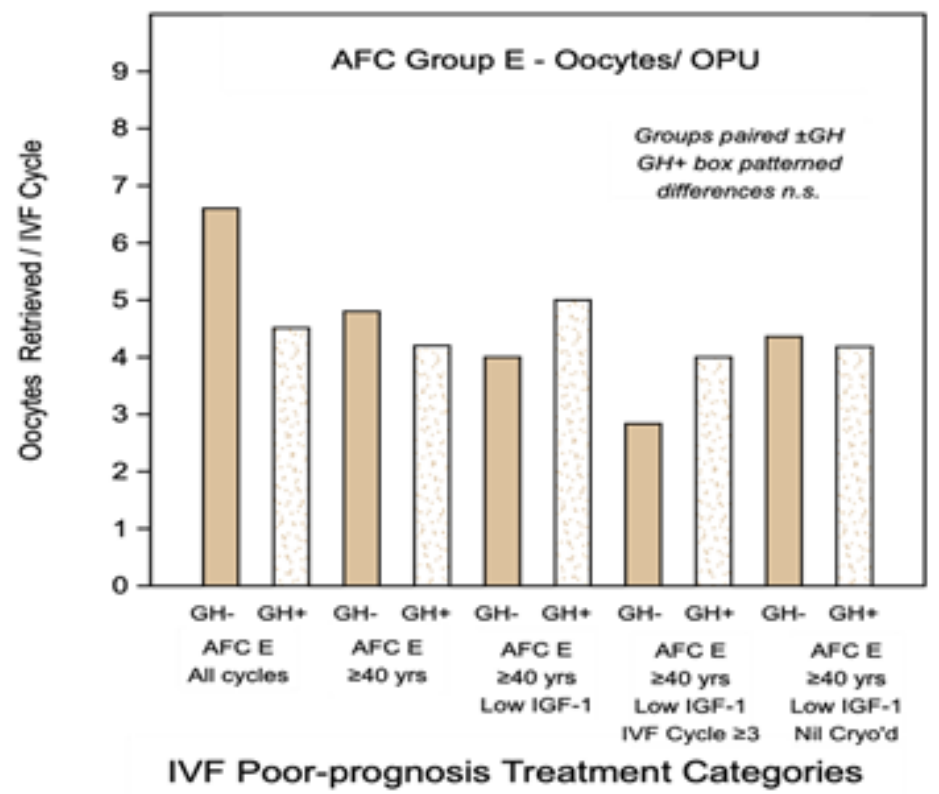

Figure 4 Oocyte recovery rates among women classified as poor prognosis who initiated IVF treatments \pm rGH

The oocyte recovery rates ranged from a mean of 9.2 for younger women ( $<35$ years) who did not receive rGH adjuvant, to a mean of 4.8 for the older women ( $\geq 40$ years). The comparable rates appeared a little lower for women who received the rGH adjuvant at 6.8 to 4.0 , but these were not significant differences. Furthermore, when the additional poor prognosis factors were analyzed, apparent minor benefits from rGH proved to be not significant (Figure 4).

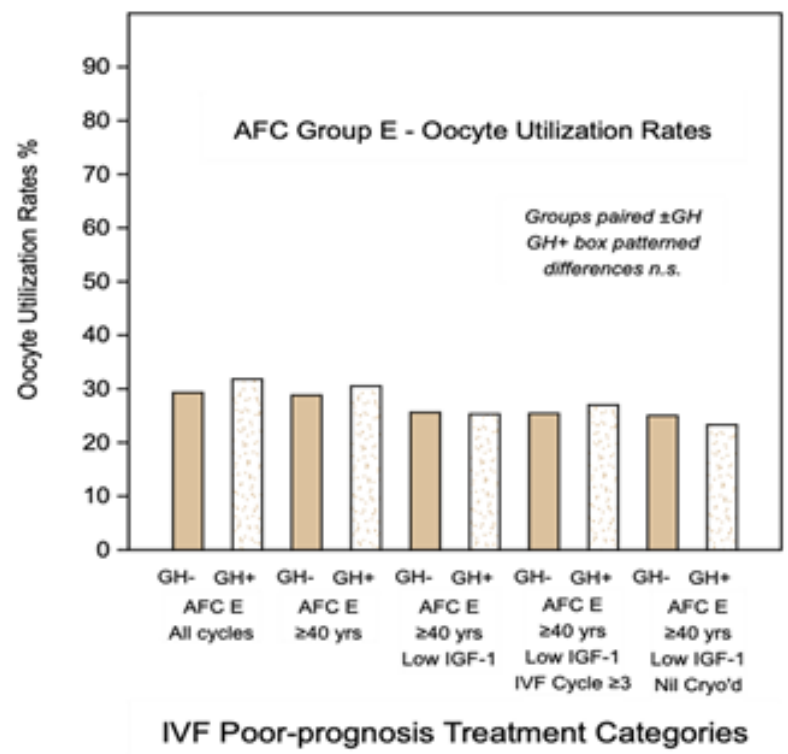

Figure 5 Oocyte utilization rates among women classified as poor prognosis who initiated IVF treatments \pm rGH

The oocyte utilization rates ranged from $31.3 \%$ in the younger women to $29.2 \%$ in the older group who did not receive $\mathrm{rGH}$. These rates were not significantly different for those women who received $\mathrm{rGH}$, ranging from $28.1 \%$ to $30.9 \%$. It was of interest that the older women showed a higher oocyte utilization rate under the influence of rGH. Furthermore, 
when the additional poor prognosis factors were analyzed, apparent minor benefits from rGH all proved to be not significant (Figure 5).

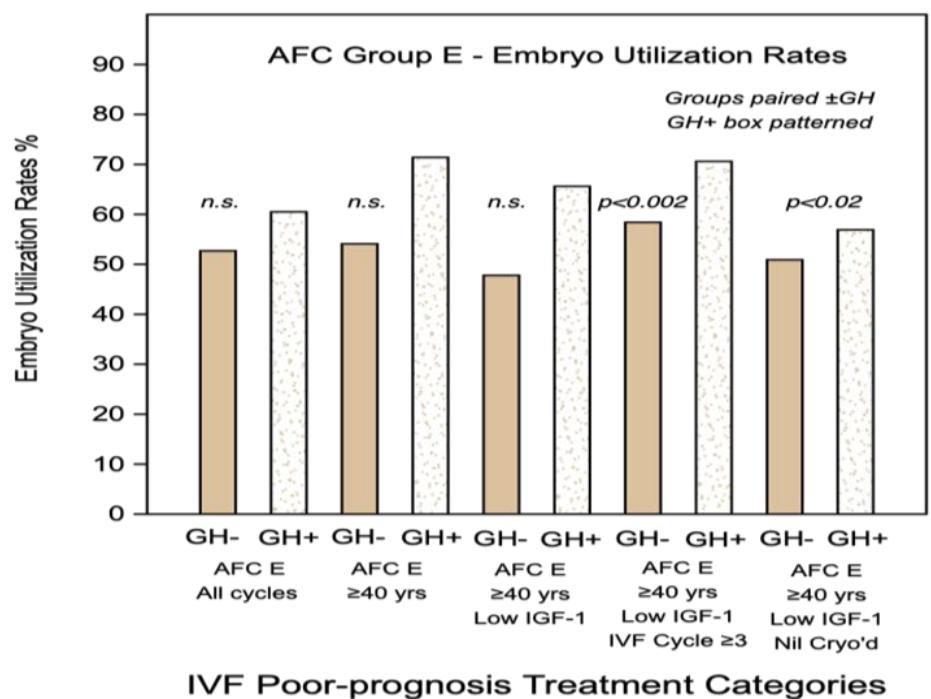

Figure 6 Embryo utilization rates among women classified as poor prognosis who initiated IVF treatments \pm rGH

The embryo utilization rates were unchanged across the age ranges for those women who did not receive rGH, being $55.4 \%$ for the younger women and $54.8 \%$ for the older group. The comparable rates for the women who received rGH ranged from $50.0 \%$ to $72.4 \%$ but this apparent benefit of $\mathrm{rGH}$ for the older women did not prove to be statistically significant in this study with limited numbers (38 women having 62 treatment cycles without rGH vs 26 women having 45 cycles with $\mathrm{rGH}$ ). However, when the additional poor prognosis factors were analyzed, higher rates of embryo utilization were detected for each group (Figure 6) with statistical significance in favor of rGH for those older women with low IGF-1 levels at higher incremental cycle $(\geq 3 ; \mathrm{p}<0.002)$ or failing to achieve any cryopreserved embryos $(\mathrm{p}<0.02)$.

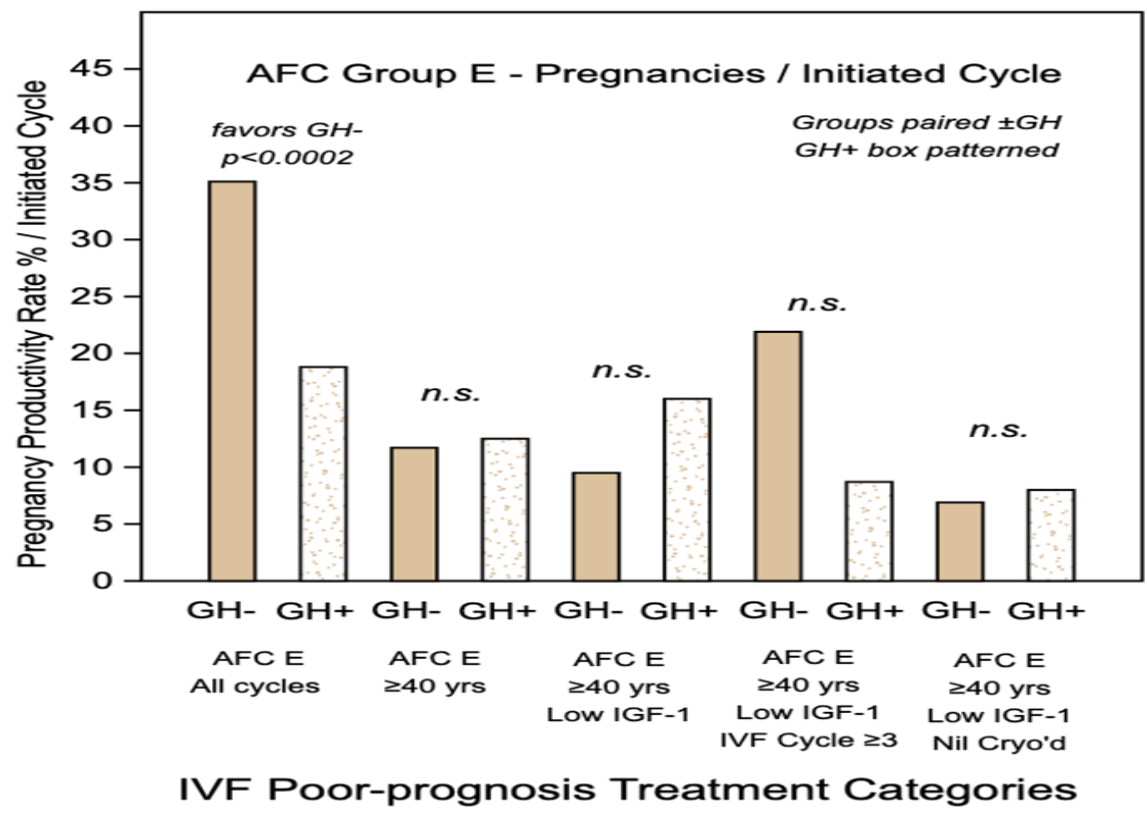

Figure 7 Pregnancy productivity rates/ initiated cycle among women classified as poor prognosis who initiated IVF treatments \pm rGH 


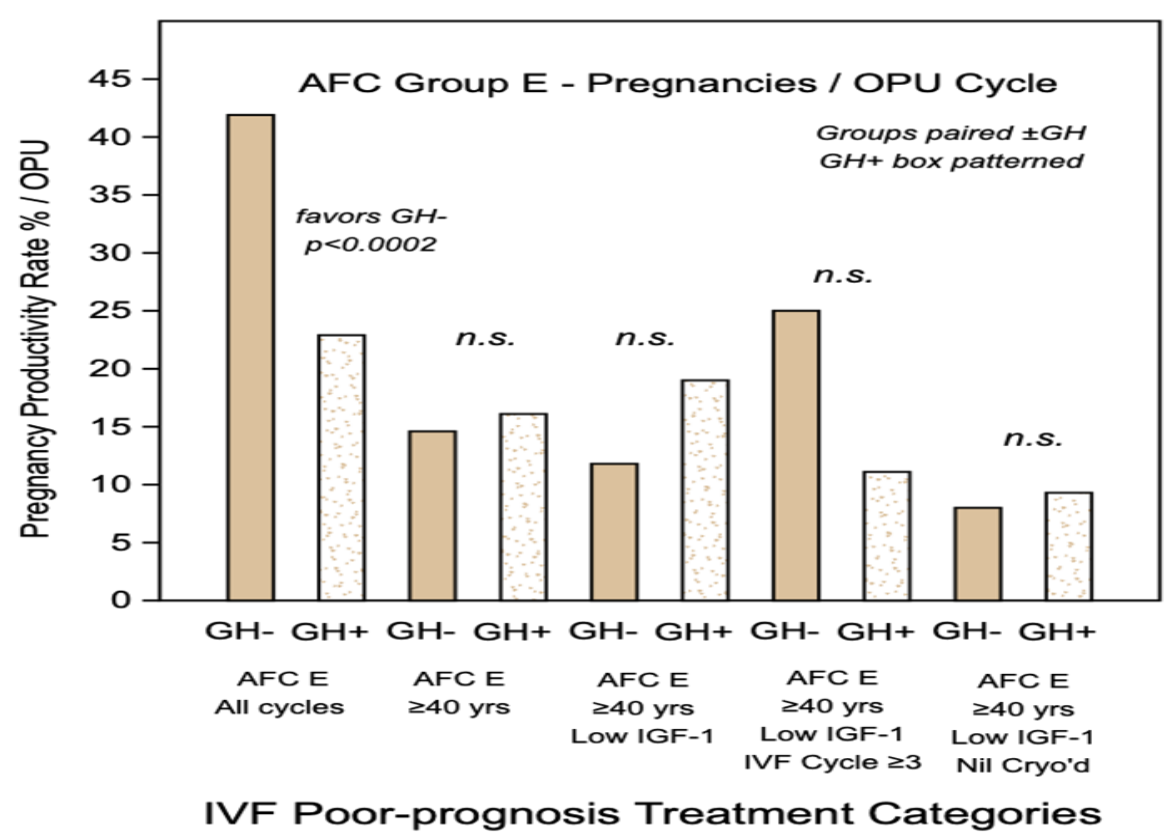

Figure 8 Pregnancy productivity rates/ OPU cycle among women classified as poor prognosis who initiated IVF treatments $\pm \mathrm{rGH}$

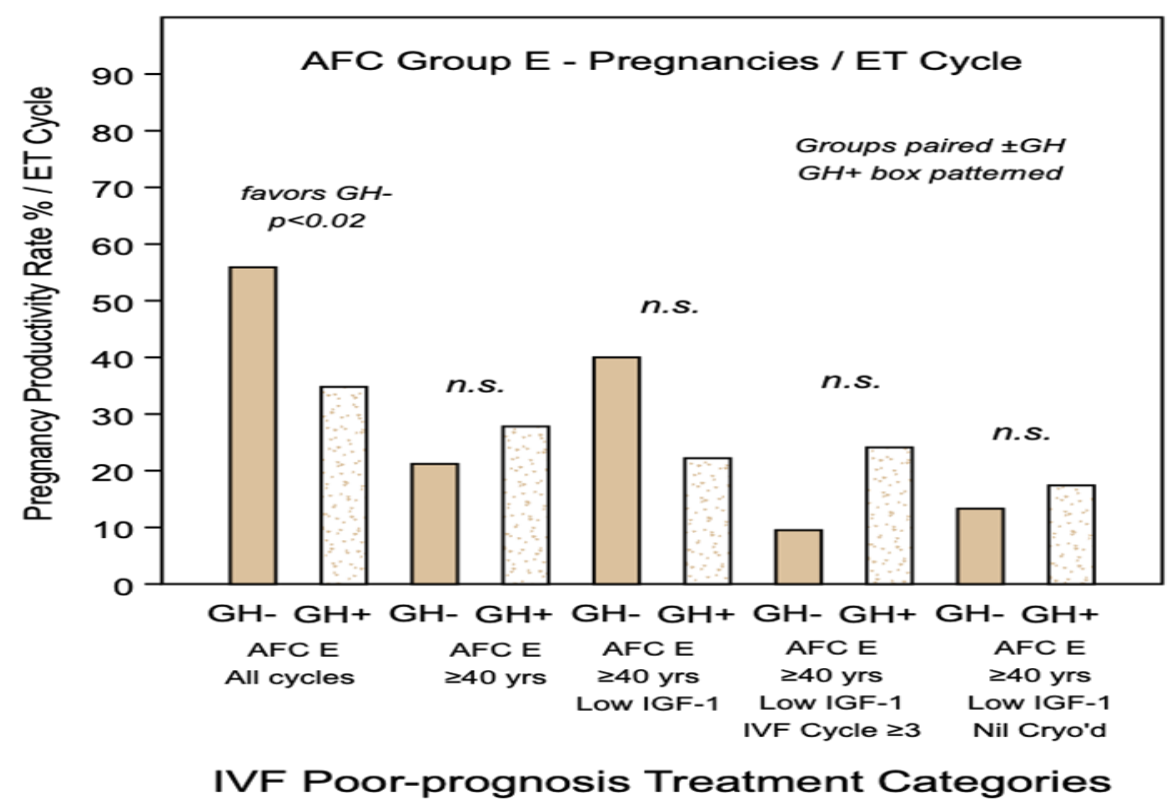

Figure 9 Pregnancy productivity rates/ ET cycle among women classified as poor prognosis who initiated IVF treatments $\pm \mathrm{rGH}$

Despite the apparent benefits noted with embryo utilization rates among the older women receiving rGH adjuvant, this did not reflect any benefits in the pregnancy productivity rates, neither for initiated cycles (Figure 7), nor for those cycles reaching OPU (Figure 8), nor for those cycles undertaking an ET procedure (Figure 9). In fact, among the younger women who did not receive $\mathrm{rGH}$, the pregnancy productivity rates were significantly higher at all stages of the IVF treatment cycle (initiated; $p<0.0002$, OPU; $p<0.0002$, ET; $p<0.02$ ). There were apparent benefits favoring $r G H$ with the additional poor prognosis factors, but none of these were statistically significant. 


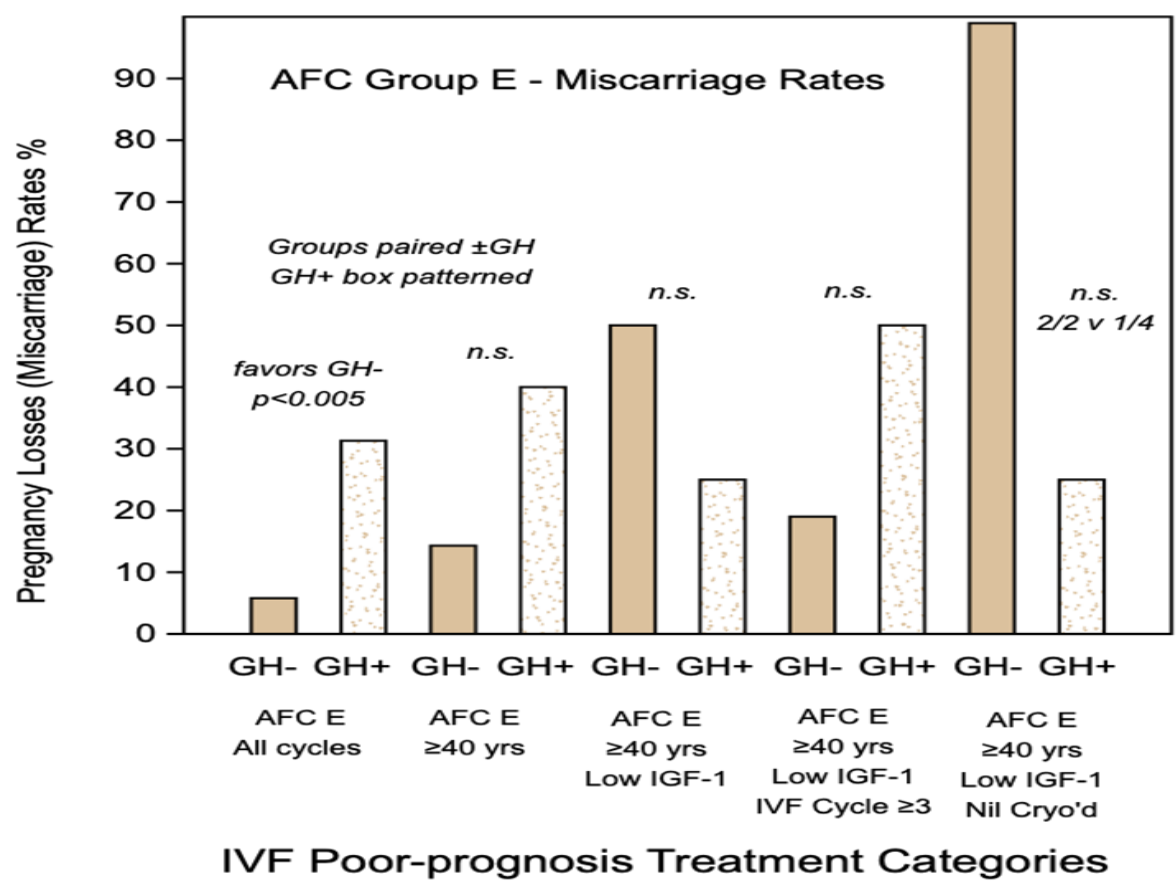

Figure 10 Miscarriage rates among women classified as poor prognosis who initiated IVF treatments \pm rGH

Miscarriage rates were stable at $15.4 \%$ in younger women to $14.3 \%$ for older women who did not receive rGH. Their overall mean rate for all ages was $17.3 \%$. However, the comparable rates for the women who received rGH adjuvant were $66.7 \%$ for the younger women and $40.0 \%$ for the older women, with an overall mean rate for all ages of $43.8 \%$. The overall miscarriage rates were significantly lower for those women not given $\mathrm{rGH}(\mathrm{p}<0.005)$ but no significant differences could be shown for the additional poor prognosis factors (Figure 10.)

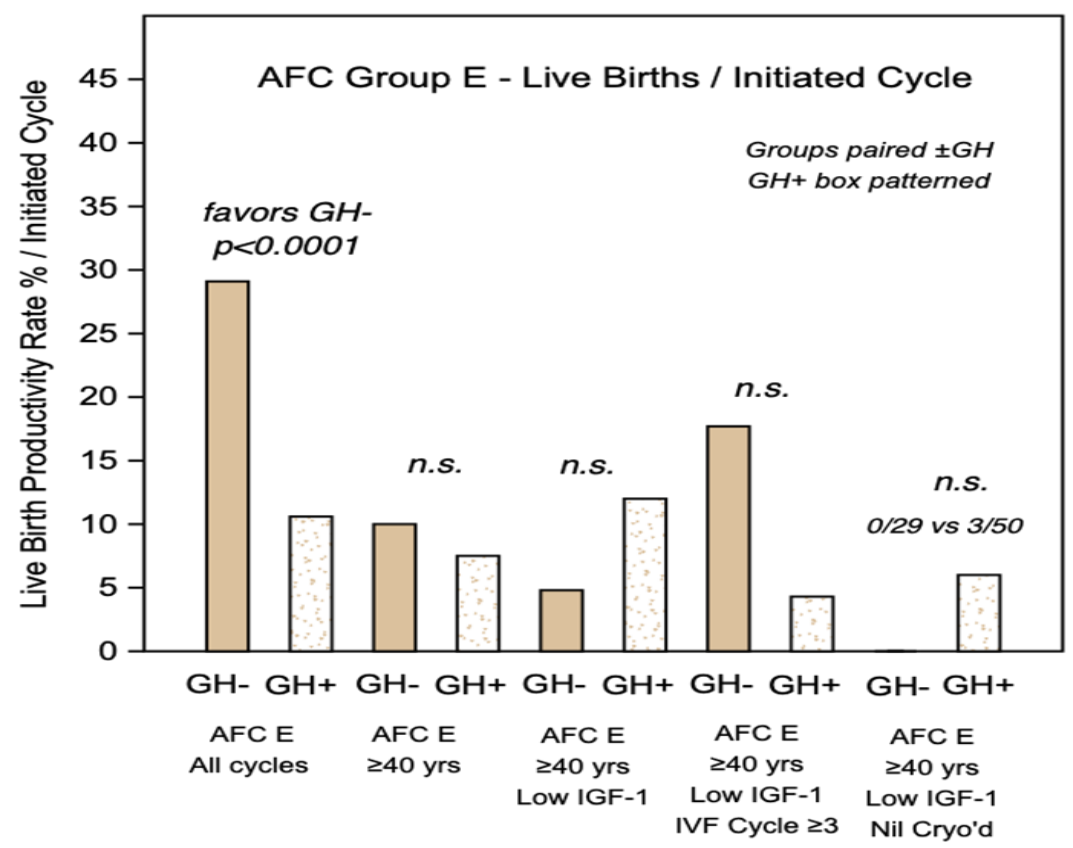

IVF Poor-prognosis Treatment Categories

Figure 11 Live birth productivity rates/ initiated cycle among women classified as poor prognosis who initiated IVF treatments $\pm \mathrm{rGH}$ 


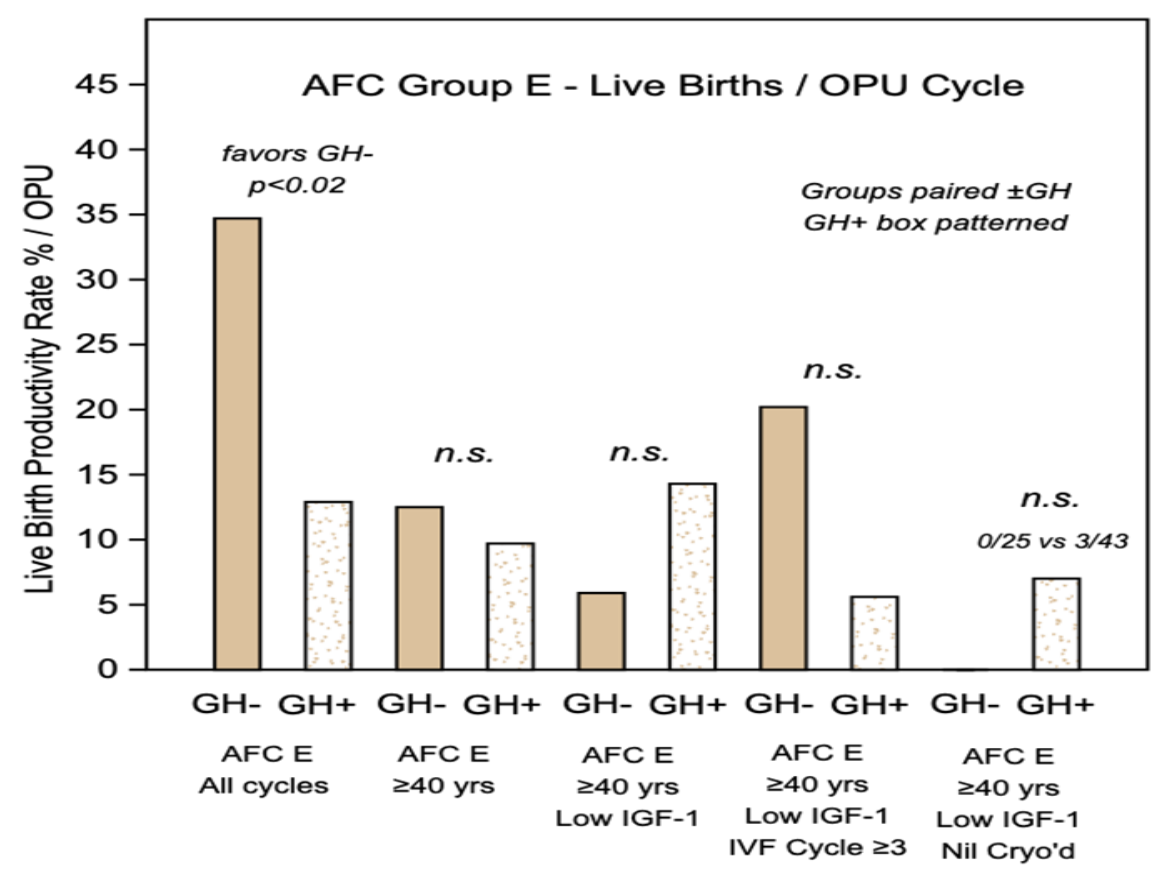

IVF Poor-prognosis Treatment Categories

Figure 12 Live birth productivity rates/ OPU cycle among women classified as poor prognosis who initiated IVF treatments $\pm \mathrm{rGH}$

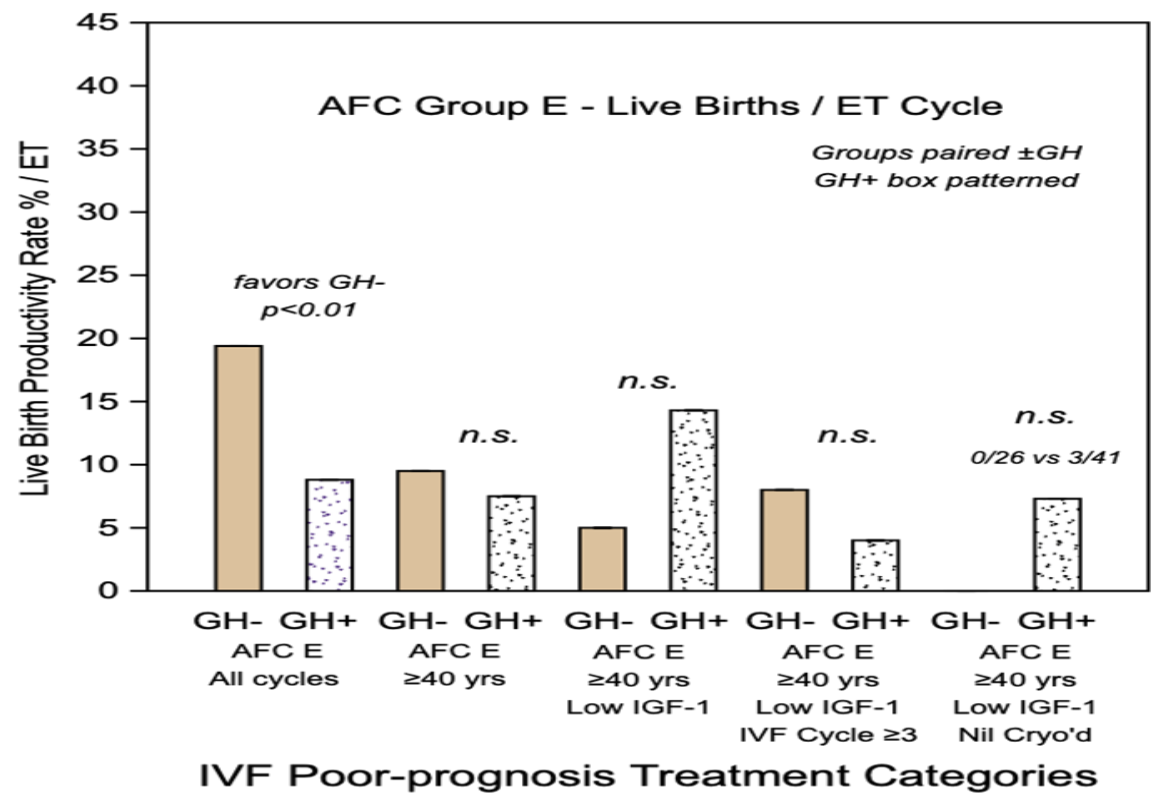

Figure 13 Live birth productivity rates/ ET cycle among women classified as poor prognosis who initiated IVF treatments $\pm \mathrm{rGH}$

Again, despite the apparent benefits shown for embryo utilization rates among the older women receiving rGH adjuvant, this did not reflect any benefits in the live birth productivity rates, neither for initiated cycles (Figure 11), nor for those cycles reaching OPU (Figure 12), nor for those cycles undertaking an ET procedure (Figure 13). In fact, among the younger women who did not receive $\mathrm{rGH}$, the live birth productivity rates were significantly higher at all stages of the IVF treatment cycle (initiated; $p<0.0001$, OPU; $p<0.02$, ET; $p<0.01$ ). There were some apparent benefits favoring $r G H$ for the additional poor prognosis factors, but none of these were statistically significant. 


\section{Discussion}

At PIVET a number of studies have been reported since 2010 which embrace the concept of poor prognosis in IVF and the potential role of $\mathrm{rGH}$ in its management [7]. The studies have been retrospective, undertaken on women who have had 3 or more IVF treatment failures. Recently, the studies have focused on the notion of adult growth hormone deficiency (AGHD) as a potential underlying cause, particularly in older women ( $\geq 40$ years) [22]. For this reason, PIVET has undertaken Assessment Cycles to determine, amongst other fertility parameters, the AFC and AMH levels to determine the ovarian reserve of women contemplating IVF, along with the IGF profile which may provide a guide to AGHD. The definitive diagnosis of AGHD would require a provocative challenge test (commonly by insulin or glucagon) and best performed by an endocrinologist. So far, PIVET patients have wanted to avoid such a time-consuming process. Hence the study reported here was intended as a prospective evaluation of the clinical outcomes of those women who are IVF-naïve and with clearly defined parameters enabling the consideration of rGH adjuvant without any previous IVF failures. It is not a randomized study and the data analysis has been performed retrospectively.

The clinical outcomes in this PIVET study which has its focus on women who have a very low ovarian reserve (Group E) can be summarized as showing no significant reduction in cancellation rates and no significant improvement in egg numbers. There is an apparent, but insignificant improvement in oocyte utilization rates and a definite improvement in embryo utilization rates for older women, which is significant when other poor prognosis factors were considered, such as repetitive IVF cycles and failure to achieve frozen embryos. This implies rGH benefits for women with poor embryo quality. However, these benefits do not flow through to significant improvements in pregnancy or live birth outcomes albeit that rGH was apparently better for older women who had additional poor prognosis factors, reflected in both pregnancy and live birth productivity rates. There is a strong note of caution in these studies which indicate that younger women have significantly better outcomes by not utilizing rGH. It also appears that rGH has no benefit in reducing miscarriage rates which were lowest across all age groups of women who did not use rGH adjuvant.

These findings are subject to a number of limitations, the main one being that the study was not set up as a prospective randomized controlled trial (RCT). Such is not feasible in PIVET's private practice setting where patients pay fees and cover the costs of all adjuvants. The numbers involved in the final analysis on the AFC group E women is approximately half that required for a definitive study (an ideal RCT would require 400 women with randomization of 200 women into each rGH arm). Such was the limitation for the LIGHT study which failed to recruit the intended numbers (only 130 of the intended 400), leaving the consideration of rGH adjuvant therapy in conjecture, although the authors claim the study provides no evidence for an improved live birth rate.

Of the 42 studies published on rGH adjuvant treatment in IVF between 2000 to 2018, almost all of the studies showed improved oocyte and embryo utilization rates, but only half demonstrated any improvement in pregnancy or live birth rates [7]. The most recent reports continue to show better oocyte and embryo quality from rGH [24] and oocytes generated under the influence of rGH have better developmental competence [25,26]. Furthermore, recent updated meta-analysis studies continue to favour rGH for increasing the chance of live births $[27,28]$.

Notwithstanding the aforementioned articles supporting rGH as an adjuvant in IVF, we are conscious of the current scepticism and criticisms concerning adjuvants in IVF [29,30]. Nonetheless, we would defend our rGH studies as a sincere attempt to improve the chance of a livebirth for those women categorized as having a poor prognosis. We agree with the editorial comments emanating from the Weill Cornell Medical College [31] that "even after the initial signal of potential efficacy 10 years ago, far too few high-quality data have been published to reassure us that growth hormone has a role in the treatment of poor responders. We as a profession should strive to conduct high-quality trials that answer our most pressing clinical questions and improve the prognosis of our most challenging patients". With these comments in mind, we acknowledge that the data arising from this, our latest, study indicates that, apart from those women with clearly defined AGHD, further studies should only focus on older women ( $\geq 40$ years) with clearly defined poor prognosis factors beyond a low AFC. Such poor prognosis factors could include a low IGF-1, repetitive failed IVF treatments ( $\geq 3$ cycles) and evidence of poor embryo quality defined by failure to achieve any embryos to cryopreservation. Furthermore, such studies can only be relevant in the circumstances of a SET policy, a commitment to blastocyst culture and an advanced vitrification technique for cryopreservation [32]. Given scientific studies supporting the role of $\mathrm{rGH}$ in improving oocyte quality generally [24,33,34], and particularly mitochondrial function [35], as well as hormonal receptor profiles on granulosa cells in older women [36], we encourage further studies to improve IVF outcomes in this group. 


\section{Conclusion}

This study failed to meet our expectations for proof that women facing poor prognosis in IVF would universally benefit from rGH adjuvant treatment. Our earlier retrospective studies reported since 2010 encouraged this expectation, however this study is structured differently as it tracks IVF naïve women (who completed an AC to define a range of poor prognosis factors) prospectively into IVF treatment, almost a quarter of whom accepted to purchase and utilize $\mathrm{rGH}$. The ensuing non-randomized data is retrospectively analyzed and shows some benefits in oocyte utilization and embryo utilization; the latter being quite significant when grouping older women with several poor prognosis factors. However, the data does not show clear benefits regarding pregnancy productivity rates nor livebirth productivity rates although this might have been related to the low numbers of older women with several poor prognosis factors. Nonetheless, a note of caution was recognized in that younger women who did not utilize rGH had significantly better pregnancy and livebirth outcomes, regardless of the number of poor prognosis factors identified. We would now adjust our advice to women seeking to improve their clinical outcomes in IVF at PIVET, dissuading the younger from rGH adjuvants, whilst encouraging older women with low ovarian reserve tests (AFC and AMH) and additional poor prognosis factors to enter larger, better designed prospective trials with either randomization or high-level case matching. Given our understanding of the role of hGH in reproduction, we would expect some women to show clear benefits; particularly if AGHD can be demonstrated. In that case the Medicare system in Australia would cover the costs of $\mathrm{rGH}$ as a replacement therapy, over a long term period if required.

\section{Compliance with ethical standards}

\section{Acknowledgments}

We are grateful for the close working relationship between PIVET Medical Centre and CLINIPATH Pathology which carried out the assays involved in the IGF profile and is also accredited by NATA.

\section{Disclosure of conflict of interest}

The entire project has been funded internally at PIVET without any external or commercial contributions. The authors declare no conflict of interest.

\section{Author Contributions}

The study was conceived by PIVET Medical Director JLY who established the data base at PIVET Medical Centre with the assistance of IT Consultant and data manager PMH. Doctors SS, MS, PR and SG are each gynecologists providing consultant services to PIVET. They have each been involved in developing the hGH adjuvant protocols and explaining the potential benefit and research investigations to the women attending PIVET for IVF-related treatments. The manuscript was written by JLY and each of the authors have read it and agreed to its content.

\section{Statement of ethical approval}

Reporting of this data was approved under Curtin University Human Ethics Committee approval no. RD_25-10 general approval for retrospective data analysis in 2010, updated in 2015, and again further updated in August 2020 for 5 years.

\section{Statement of informed consent}

PIVET is accredited with both the self-regulatory National Australian Reproductive Technology Committee (RTAC) as well as the Reproductive Technology Council (RTC) of Western Australia. Consent forms received approval under both regulatory bodies. The assay laboratory is accredited on an annual basis by the National Australian Testing Authority (NATA). All the adjuvants mentioned in this article were provided under informed consent with women providing written approval, witnessed by an independent person. The specific information and consent form for recombinant human growth hormone (hGH) was shown in Figure 2.

\section{References}

[1] Newman JE, Paul RC, Chambers GM. Assisted reproductive technology in Australia and New Zealand 2018. Sydney: National Perinatal Epidemiology and Statistics Unit, the University of New South Wales, Sydney. 2020; 1-83. 
[2] Yovich JL, Zaidi S, Nguyen MDK, Hinchliffe PM. Measuring IGF-1 and IGFBP-3 profiles in women seeking assisted reproduction; relationship to clinical parameters (Study 1). J Pers Med. 2020; 10(3): 122, 1-15.

[3] Yovich JL, Zaidi S, Nguyen MDK, Hinchliffe PM. Measuring IGF-1 and IGFBP-3 profiles in women seeking assisted reproduction; relationship to ovarian reserve parameters (Study 2). GSC Biol \& Pharmaceutical Sciences. 2020; 13(02): 035-053.

[4] Yovich JL, Zaidi S, Nguyen MDK, Hinchliffe PM. Measuring IGF-1 and IGFBP-3 profiles in women seeking assisted reproduction; relationship to serum growth hormone levels (Study 3). GSC Biol \& Pharmaceutical Sciences. 2020; 13(03): 032-053.

[5] Yovich JL, Zaidi S, Nguyen MDK, Hinchliffe PM. Measuring IGF-1 and IGFBP-3 profiles in women seeking assisted reproduction; response of women categorised as poor prognosis to growth hormone adjuvant therapy (Study 4). GSC Biol \& Pharmaceutical Sciences. 2020; 13(03): 064-078.

[6] Yovich JL, Zaidi S, Nguyen MDK, Hinchliffe PM. Measuring IGF-1 and IGFBP-3 profiles in women seeking assisted reproduction; relevance to clinical outcomes from in vitro fertilization (Study 5). GSC Biol \& Pharmaceutical Sciences. 2020; 13(03): 079-096.

[7] Yovich JL, Ye Y, Regan SLP, Keane KN. The evolving concept of poor-prognosis for women undertaking IVF and the notion of growth hormone as an adjuvant; a single-center viewpoint. Front Endocrinol. 2019; 10(808): 1-14.

[8] Yovich JL, Craft IL. Founding pioneers of IVF: Independent innovative researchers generating livebirths within 4 years of the first birth. Reprod Biol. 2018; 18: 317-323.

[9] Yovich JL. Founding pioneers of IVF Update: Independent innovative researchers generating livebirths within 4 years of the first birth. Reprod Biol. 2020; 20: 111-113.

[10] Yovich JL. How to Prepare the Egg and Embryo to Maximise IVF Success. In: Monitoring the stimulated IVF cycle. Section II: Stimulation for IVF (Eds: Gabor T Kovacs, Anthony J Rutherford, David K Gardner). Cambridge University Press, Cambridge. 2019; 94-120.

[11] Yovich J, Stanger J, Hinchliffe P. Targeted gonadotrophin stimulation using the PIVET algorithm markedly reduces the risk of OHSS. Reprod Biomed Online. 2012; 24(3): 281-292.

[12] Yovich JL, Alsbjerg B, Conceicao JL, Hinchliffe PM, Keane KN. PIVET rFSH dosing algorithms for individualized controlled ovarian stimulation enables optimized pregnancy productivity rates and avoidance of ovarian hyperstimulation syndrome. Drug Des Devel Ther. 2016; 10: 2561-2573.

[13] Yovich JL, Hinchliffe PM, Lingam S, Srinivasan S, Keane KN. Adjusting the PIVET rFSH dosing algorithm for the biosimilar Bemfola product. J Fertil In vitro IVF Worldw Reprod Med Genet Stem Cell Biol. 2018; 5: 3.

[14] Kuwayama M, Vajta G, Kato O, Leibo SP. Highly efficient vitrification method for cryopreservation of human oocytes. Reprod Biomed Online. 2005; 11: 300-308.

[15] Yovich JL, Conceicao JL, Marjanovich N, Ye Y, Hinchliffe PM, Dhaliwal SS, Keane KN. An ICSI rate of 90\% minimizes complete failed fertilization and provides satisfactory implantation rates without elevating fetal abnormalities. Reprod Biol. 2018; 18: 301-311.

[16] Keane K, Cruzat VF, Wagle S, Chaudhary N, Newsholme P, Yovich J. Specific ranges of anti-Mullerian hormone and antral follicle count correlate to provide a prognostic indicator for IVF outcome. Reprod Biol. 2017; 17: 51-59.

[17] Mustafa KB, Keane KN, Walz NL, Mitrovic KI, Hinchliffe PM, Yovich JL. Live Birth Rates are satisfactory following multiple IVF treatment cycles in poor prognosis patients. Reprod Biol. 2017; 17: 34-41.

[18] Yovich JL, Conceicao J, Hinchliffe P, Keane K. Which blastocysts should be considered for genetic screening? Hum Reprod. 2015; 30: 1743-1745.

[19] Yovich JL. Stanger JD, Keane KN. Cumulative live birth rate: An outmoded term. JFIV Reprod Med Genet. 2016; 4: 165.

[20] Hammer $\emptyset$, Harper DAT, Ryan PD. PAST: Paleontological Statistics software package for education and data analysis. Palaentologia Electronica. 2001; 4(1): 1-9.

[21] Yovich JL, Regan SL, Zaidi SN, Keane KN. The concept of growth hormone deficiency affecting clinical prognosis in IVF. Front Endocrinol. 2019; 10(650): 1-9. 
[22] Norman RJ, Alvino H, Hull LM, Mol BW, Hart RJ, Kelly T-L, et al. Human growth hormone for poor responders: a randomized placebo-controlled trial provides no evidence for improved live birth rate. Reprod Biomed Online. 2019; 38: 908-15.

[23] Tesarik J, Galán-Lázaro M, Conde-López C, Chiara-Rapisarda AM, Mendoza-Tesarik R. The effect of GH administration on oocyte and zygote quality in young women with repeated implantation failure after IVF. Front Endocrinol. 2020; 11; 519-572, 1-7.

[24] Keane KN, Ye Y, Regan SLP, Dhaliwal SS, Yovich JL. Live birth outcomes of vitrified embryos generated under growth hormone stimulation are improved for women categorized as poor-prognosis. Clin Exp Reprod Med. 2019; 46(40): 178-188.

[25] Li J, Chen Q, Wang J, Huang G, Ye H. Does growth hormone supplementation improve oocyte competence and IVF outcomes in patients with poor embryonic development? A randomized controlled trial. BMC Pregnancy and Childbirth. 2020; 20(310): 1-10.

[26] Zhang Y, Zhang C, Shu J, Guo J, Chang H-M, Leung PCK, Sheng J-Z, Huang H. Adjuvant treatment strategies in ovarian stimulation for poor responders undergoing IVF: a systematic review and network meta-analysis. Hum Reprod Update. 2020; 26(2): 247-263.

[27] Yang P, Wu R, Zhang $\mathrm{H}$. The effect of growth hormone supplementation in poor ovarian responders undergoing IVF or ICSI: a meta-analysis of randomized controlled trials. Reprod Biol Endocrinol. 2020; 18(1): 76, 1-10.

[28] Farquhar C. Add-ons for assisted reproductive technology: can we be honest here? Fertil Steril. 2019; 112(6): 971-972.

[29] Annual Capri Workshop Group. IVF, from the past to the future: the inheritance of the Capri Workshop Group. Hum Reprod Open. 2020; 3: 1-9.

[30] Bortoletto P, Spandorfer S. Growth hormone: in search of the holy grail for poor responders (or a felony). Fertil Steril. 2020; 114(1): 63-64.

[31] Yovich JL, Ye Y, Keane KN. Growth hormone adjuvant trial for poor responders undergoing IVF. Eur J Obstet Gynecol. 2019; 236: 249-251.

[32] Devesa J, Caicedo D. The role of growth hormone on ovarian functioning and ovarian angiogenesis. Front Endocrinol. 2019; 10: 1-16.

[33] Ipsa E, Cruzat VF, Kagize JN, Yovich JL, Keane KN. Growth Hormone and Insulin-like growth factor in reproductive tissues. Front Endocrinol. 2019; 777: 1-14.

[34] Weall BM, Al-Samerria S, Conceicao J, Yovich JL, Almahbobi G. A direct action for GH in improvement of oocyte quality in poor-responder patients. Reproduction. 2015; 149: 147-154.

[35] Regan SLP, Knight PG, Yovich JL, Arfuso F, Dharmarajan A. Growth hormone during in vitro fertilization in older women modulates the density of receptors in granulosa cells, with improved pregnancy outcomes. Fertil Steril. 2018; 110: 1298-1310.

\section{Author's short biography}

\begin{tabular}{|l} 
Professor Dr. John Yovich MD PhD FRCOG FRANZCOG CREI graduated, from the University \\
of Western Australia in 1970, progressing into Specialist O\&G practice in 1976. Following \\
laboratory research and clinical work over 4-years in London 1976-80 with Professor Ian \\
Craft, John presented his PhD thesis “Human pregnancies achieved by in-vitro fertilization" \\
(UWA 1985). He established PIVET Medical Centre in 1981, generating WA's first IVF child in \\
July 1982. John has assisted many IVF clinics to establish worldwide during the 1980's and, \\
at age 75 years, maintains a full-time clinical program for fertility-related gynecological \\
disorders as well as IVF. His academic work is now conducted through the Department of \\
Pharmacy and Biomedical Sciences at Curtin University.
\end{tabular}

